\title{
Effect of water current on some growth performance and water quali- ty in a closed rainbow trout (Oncorhynchus mykiss) culture system
}

\author{
M Mohamadizadeh $\mathrm{Kh}^{1}$, M Shamsaie $\mathbf{M}^{1}$, Y Abdollahtabar ${ }^{1}$ and A Soltani² \\ ${ }^{1}$ Aquaculture Department, Faculty of Agriculture and Natural Resource, Islamic Azad University, Science and \\ Research Branch of Tehran, Tehran, Iran \\ ${ }^{2}$ Department of Irrigation and Reclamation engineering, Faculty of Agricultural Engineering and Technology, \\ University of Tehran, Iran
}

Received: January 2014 Accepted: April 2014

\begin{abstract}
The effects of water current increment on rainbow trout (Oncorhynchus mykiss) culture were investigated during a 35 day experiment. Fish weighing $5.5 \mathrm{~g}$ were stocked in aquarium tanks. Four different water speeds $(0,3.5,7,10.5 \mathrm{~cm} / \mathrm{s})$ were provided for each treatment in three replicates. These different currents water were provided by reusing outlet water of each culture unit. Length, weight, daily growth rate (DGR), specific growth rate (SGR), condition factor (CF) and survival rate (SR) were evaluated in each treatment. Also, the changes of $\mathrm{NO} 2, \mathrm{NO} 3$, $\mathrm{NH} 3, \mathrm{NH} 4+$, total hardness and $\mathrm{pH}$ were measured. The data variance analysis showed significant differences among all treatments during the first week $(\mathrm{P}<0.01)$. However, these results were not observed in following days. Based on Duncan's test results, the best survival rate $(97 \%)$, daily growth rate (1), SGR $(6 \%)$ and average weight $(24 \mathrm{~g})$ were achieved in $10.5 \mathrm{~cm} / \mathrm{s}$.
\end{abstract}

Keywords: current speed, closed system, outlet water, rainbow trout, Iran.

\section{Introduction}

During the last decade, rainbow trout (Oncorhynchus mykiss) production has been expanded in

Correspondence M Shamsaie M, Aquaculture Department, Faculty of Agriculture and Natural Resource, Islamic Azad University, Science and Research Branch of Tehran, Tehran, Iran (e-mail: drshamsaie@gmail.com)
Iran and its amount grew from $9000 \mathrm{mt}$ in 2000 to $130000 \mathrm{mt}$ in 2014. Aquaculture increment could be considered as one of the main reasons for water pollution in the world. Thus, water quality protection in fish culture is important and this leads industry to use modern systems such as recirculation aquaculture system (RAS) in order to get maximum production without polluting water. The possibility of outlet water refining is the main advantage of these systems and $\mathrm{pH}$, temperature and control of bacterial disease are their other advantages (Willoughby 1999). However, they are very expensive. But what would be happened if some functions of RAS are eliminated and water velocity is increased through the culture unit, larger fish can stand against more water velocity (Sedgwick 1990). Therefore, this experiment was undertaken to evaluate the effect of water velocity, physical filtration and aeration in a closed rainbow trout culture system. The study was run via a randomize complete blocks design with 4 treatments in 3 replicates. Different water velocities of $0,3.5,7,10.5 \mathrm{~cm} / \mathrm{s}$ along with twelve plastic aquariums $(200 \times 40 \times 15 \mathrm{~cm})$ were considered.

\section{Materials and Methods}

The experiment was carried out during a period of 35 days in Khojir Natural Resources Station, Tehran, Iran. Each plot contained $60 \mathrm{~L}$ of water with a Renault air pump for aerating. Rainbow trout weighing $5.5 \mathrm{~g}$ were provided from a private farm in Semnan province. Twelve fish were introduced to each aquarium after $48 \mathrm{~h}$ adaptation and feeding was started $48 \mathrm{~h}$ after fish transportation. Water recircu- 


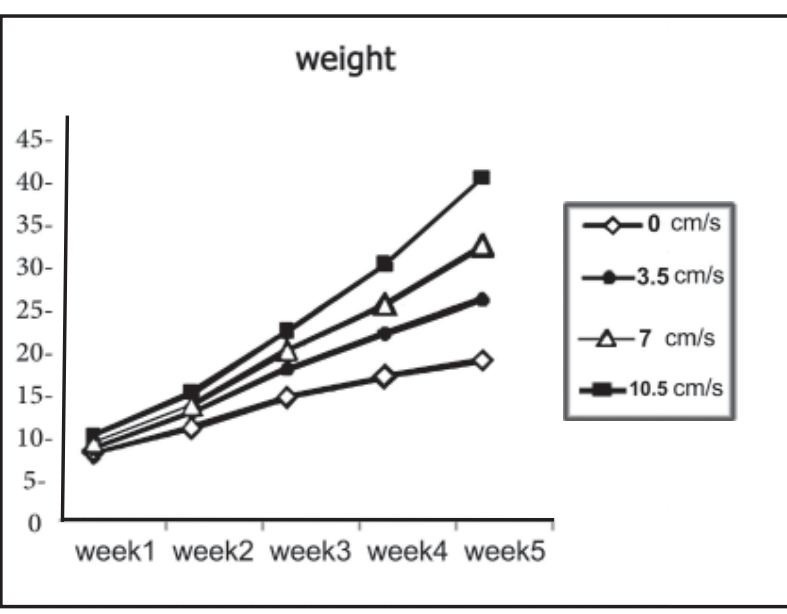

Figure 1 Changes of fry weight in different treatments during the experiment period.

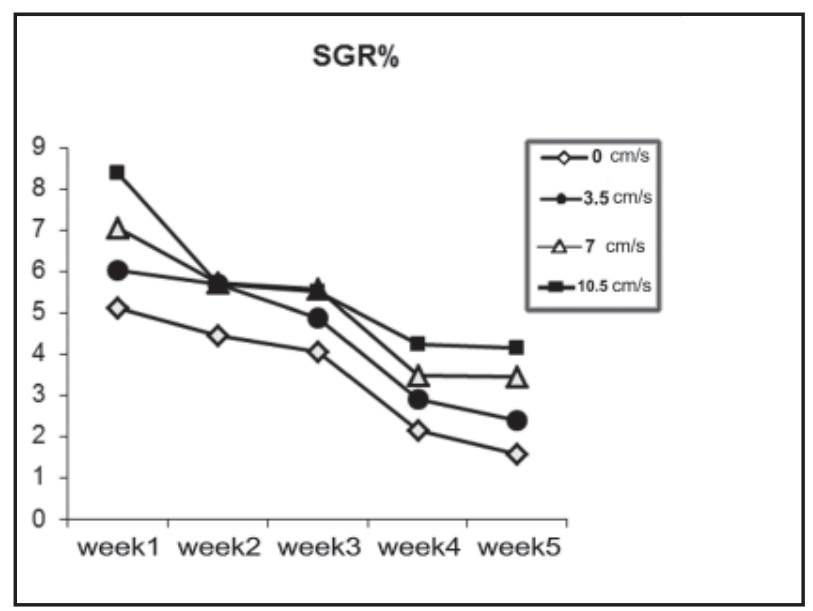

Figure 3 Changes of fry SGR\% in different treatments during the experiment period.

lation was performed by external electrical pumps via each plot which was connected to $30 \mu$ mesh size filter bags in order to physically filter water. These filter bags were cleaned manually daily, using fresh water. Evaporated water in each plot was replaced by isotherm fresh water. The water was gathered from the bottom of each plot by pumping and recirculating to aquarium after filtering. Fish stocking was considered $200 / \mathrm{m} 2$, which was more than two fold Iranian farms average density. Fish were fed with a commercial food (Biomar Company, France) at 4\% body weight (Jeffrey 1999) 5 times a day between 7 am and $7 \mathrm{pm}$. Feed comprised of $54 \%$ crude protein, $18 \%$ crude fat, $0.5 \%$ fiber, $10 \%$ ash and $1.4 \%$ phosphor. Water temperature and $\mathrm{pH}$ were measured daily during the experiment. Also, fry weight, length, specific growth rate (SGR) daily growth rate (DGR) survival rate (SR) and condition

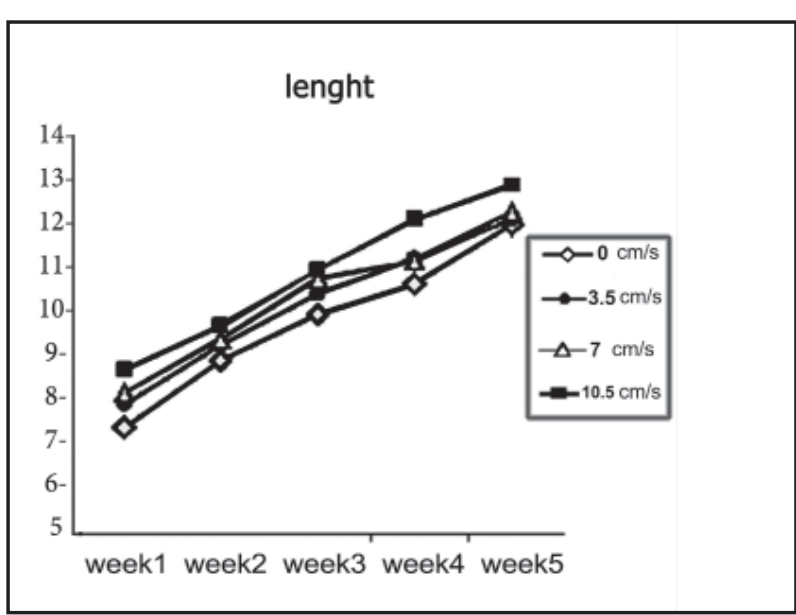

Figure 2 Changes of fry length in different treatments during the experiment period.

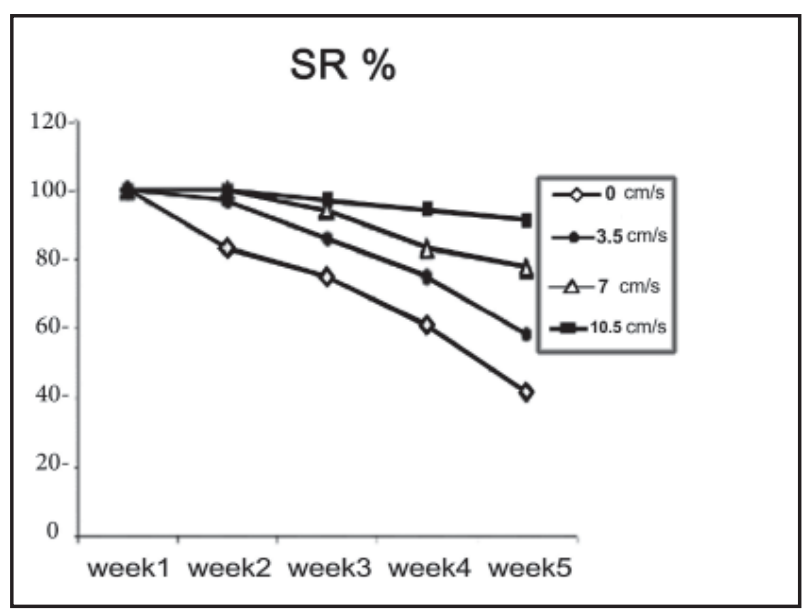

Figure 4 Changes of fry SR $\%$ in different treatments during the experiment period.

factor $(\mathrm{CF})$ were weekly measured using below equations (Castell \& Tiews 1980):

DGR: [(final weight (gr) - initial weight (gr) ) $\div$ experiment days $] \times 100$

SGR: [(Ln final weight- Ln initial weight $) \div$ experiment days $] \times 100$

CF: (weight $(\mathrm{g}) \div$ lenght 3$) \times 100$

SR: (live fry $\div$ total plot fry $) \times 100$

Data were analysed by SPSS software, version 14 and Duncan's averages comparing test was used to determine the best fish indexes averages among different treatments.

\section{Results}

Average oxygen demand was $7,7.5,8.5,10.5$ in 0 , $3.5,7$ and $10 \mathrm{~cm} / \mathrm{s}$ treatments, respectively. Table 1 shows significant difference for measured variables 
Iranian Journal of Aquatic Animal Health

Table1 Growth performance of trout treated in different water qualities one week post-experiment at $18 \pm 1.19^{\circ} \mathrm{C}$

\begin{tabular}{llllll}
\hline Growth factor & Treatment & \multicolumn{5}{l}{} \\
\cline { 2 - 6 } & F5 & T1 & T2 & T3 & T4 \\
\hline Weight $(\mathrm{g})$ & $* * 16.823$ & $7.8731^{\mathrm{c}} \pm 0.30$ & $8.3867^{\mathrm{b}} \pm 0.38$ & $9.0191^{\mathrm{b}} \pm 0.23$ & $9.8938^{\mathrm{a}} \pm 0.42$ \\
Length $(\mathrm{cm})$ & $* * 11.173$ & $7.4961^{\mathrm{c}} \pm 0.17$ & $8.0231^{\mathrm{bc}} \pm 0.48$ & $8.3046^{\mathrm{ab}} \pm 0.18$ & $8.8276^{\mathrm{a}} \pm 0.18$ \\
SGR\% & $* * 16.892$ & $5.1172^{\mathrm{c}} \pm 0.55$ & $6.0173^{\mathrm{b}} \pm 0.65$ & $7.0590^{\mathrm{b}} \pm 0.53$ & $8.3791^{\mathrm{a}} \pm 0.62$ \\
DGR\% & $* * 16.814$ & $0.3389^{\mathrm{c}} \pm 0.04$ & $0.4123^{\mathrm{bc}} \pm 0.05$ & $0.5026^{\mathrm{b}} \pm 0.04$ & $0.6276^{\mathrm{a}} \pm 0.06$ \\
CF\% & $* * 6.608$ & $1.8666^{\mathrm{a}} \pm 0.10$ & $1.6381^{\mathrm{b}} \pm 0.21$ & $1.5751^{\mathrm{b}} \pm 0.04$ & $1.4381^{\mathrm{b}} \pm 0.02$ \\
SR\% & $3.27^{\mathrm{ns}}$ & $100.000^{\mathrm{a}} \pm 0$ & $100.000^{\mathrm{a}} \pm 0$ & $100.000^{\mathrm{a}} \pm 0$ & $100.000^{\mathrm{a}} \pm 0$ \\
\hline
\end{tabular}

** Significant differences in $\mathrm{P}<0.01,{ }^{\mathrm{ns}}$ non significant differences, $\mathrm{T}=$ Treatment.

Table 2 Growth performance of trout treated in different water qualities two week post-experiment at $18 \pm 1.19^{\circ} \mathrm{C}$

\begin{tabular}{|c|c|c|c|c|c|}
\hline \multirow[t]{2}{*}{ Studied indicators } & \multicolumn{5}{|c|}{ Treatment } \\
\hline & F5 & $\mathrm{T} 1$ & $\mathrm{~T} 2$ & $\mathrm{~T} 3$ & $\mathrm{~T} 4$ \\
\hline Weight (gr) & $9.162 * *$ & $10.7598^{\mathrm{c}} \pm 0.67$ & $12.5396^{\mathrm{bc}} \pm 0.32$ & $13.4600^{\mathrm{ab}} \pm 0.70$ & $14.7584^{\mathrm{a}} \pm 0.98$ \\
\hline Length (cm) & $1.531^{\mathrm{ns}}$ & $9.0186^{\mathrm{a}} \pm 0.75$ & $9.3664^{\mathrm{a}} \pm 0.46$ & $9.5305^{\mathrm{a}} \pm 0.28$ & $9.8107^{\mathrm{a}} \pm 0.17$ \\
\hline SGR $\%$ & $3.884^{*}$ & $4.4502^{b} \pm 0.55$ & $5.7005^{\mathrm{a}} \pm 0.86$ & $5.7027^{a} \pm 0.22$ & $5.7131^{\mathrm{a}} \pm 0.33$ \\
\hline DGR $\%$ & $5.600^{*}$ & $0.4095^{\mathrm{b}} \pm 0.07$ & $0.5932^{\mathrm{a}} \pm 0.13$ & $0.6341^{\mathrm{a}} \pm 0.05$ & $0.6949^{\mathrm{a}} \pm 0.07$ \\
\hline $\mathrm{CF} \%$ & $0.115^{\text {ns }}$ & $1.4943^{\mathrm{a}} \pm 0.29$ & $1.5242^{\mathrm{a}} \pm 0.06$ & $1.5558^{\mathrm{a}} \pm 0.06$ & $1.5616^{\mathrm{a}} \pm 0.05$ \\
\hline $\mathrm{SR} \%$ & $8.296 * *$ & $83.300^{\mathrm{b}} \pm 8.3$ & $97.200^{\mathrm{a}} \pm 4.84$ & $100.000^{\mathrm{a}} \pm 0$ & $100.000^{\mathrm{a}} \pm 0$ \\
\hline
\end{tabular}

** Significant differences in $\mathrm{P}<0.01$, ns non significant differences, $\mathrm{T}=$ Treatment.

Table 3 Growth performance of trout treated in different water qualities three week post-experiment at $18 \pm 1.19^{\circ} \mathrm{C}$

\begin{tabular}{|c|c|c|c|c|c|}
\hline \multicolumn{6}{|c|}{ Studied indicators } \\
\hline \multirow[t]{2}{*}{ F5 } & \multicolumn{5}{|l|}{ Treatment } \\
\hline & & $\mathrm{T} 1$ & $\mathrm{~T} 2$ & T3 & $\mathrm{T} 4$ \\
\hline Weight (gr) & $6.783^{* *}$ & $14.3287^{\mathrm{c}} \pm 1.71$ & $17.7351^{\mathrm{bc}} \pm 2.80$ & $19.8966^{\mathrm{ab}} \pm 1.87$ & $22.2434^{\mathrm{a}} \pm 2.39$ \\
\hline Length (cm) & $8.033^{* *}$ & $10.0897^{\mathrm{c}} \pm 0.40$ & $10.5445^{\mathrm{bc}} \pm 0.21$ & $10.9010^{\mathrm{ab}} \pm 0.20$ & $11.0927^{a} \pm 0.20$ \\
\hline $\mathrm{SGR} \%$ & $1.852 \mathrm{~ns}$ & $4.0416^{\mathrm{a}} \pm 0.82$ & $4.8838^{\mathrm{a}} \pm 0.83$ & $5.4926^{\mathrm{a}} \pm 0.59$ & $5.5548^{\mathrm{a}} \pm 1.20$ \\
\hline DGR\% & $5.057^{*}$ & $0.5097^{b} \pm 0.14$ & $0.7421^{\mathrm{ab}} \pm 0.21$ & $0.9194^{\mathrm{a}} \pm 0.16$ & $1.0692^{\mathrm{a}} \pm 0.20$ \\
\hline $\mathrm{CF} \%$ & $3.286 \mathrm{~ns}$ & $1.3903^{\mathrm{b}} \pm 0.009$ & $1.5054^{\mathrm{ab}} \pm 0.14$ & $1.5329^{\mathrm{ab}} \pm 0.06$ & $1.6252^{\mathrm{a}} \pm 0.08$ \\
\hline $\mathrm{SR} \%$ & $5.735^{*}$ & $74.967^{\mathrm{b}} \pm 8.35$ & $86.067^{\mathrm{ab}} \pm 9.58$ & $94.400^{\mathrm{a}} \pm 4.84$ & $97.200^{\mathrm{a}} \pm 4.84$ \\
\hline
\end{tabular}

** Significant differences in $\mathrm{P}<0.01,{ }^{\mathrm{ns}}$ non significant differences, $\mathrm{T}=$ Treatment.

Table 4 Growth performance of trout treated in different water qualities four week post-experiment at $18 \pm 1.19^{\circ} \mathrm{C}$

\begin{tabular}{|c|c|c|c|c|c|}
\hline \multirow[t]{2}{*}{ Studied indicators } & \multicolumn{5}{|c|}{ Treatment } \\
\hline & F5 & T1 & $\mathrm{T} 2$ & T3 & T4 \\
\hline Weight (gr) & $6.850^{* *}$ & $16.7387^{\mathrm{c}} \pm 2.78$ & $21.8137^{\mathrm{bc}} \pm 4.91$ & $25.4217^{\mathrm{ab}} \pm 3.27$ & $29.9999^{\mathrm{a}} \pm 4.36$ \\
\hline Length (cm) & $6.636^{* *}$ & $10.7669^{b} \pm 0.33$ & $11.3195^{\mathrm{b}} \pm 0.48$ & $11.3698^{b} \pm 0.32$ & $12.2400^{\mathrm{a}} \pm 0.46$ \\
\hline SGR $\%$ & $6.922 * *$ & $2.1568^{c} \pm 0.67$ & $2.8973^{\mathrm{bc}} \pm 0.51$ & $3.4641^{\mathrm{ab}} \pm 0.49$ & $4.2246^{\mathrm{a}} \pm 0.60$ \\
\hline DGR \% & $6.808^{* *}$ & $0.3442^{\mathrm{c}} \pm 0.15$ & $0.5826^{\mathrm{bc}} \pm 0.19$ & $0.7892^{\mathrm{ab}} \pm 0.20$ & $1.1080^{\mathrm{a}} \pm 0.28$ \\
\hline $\mathrm{CF} \%$ & $9.790 * *$ & $1.3323^{\mathrm{c}} \pm 0.10$ & $1.4724^{\mathrm{bc}} \pm 0.13$ & $1.6270^{\mathrm{ab}} \pm 0.08$ & $1.7460^{\mathrm{a}} \pm 0.05$ \\
\hline $\mathrm{SR} \%$ & $6.016^{* *}$ & $61.100^{c} \pm 12.73$ & $74.967^{\mathrm{bc}} \pm 8.35$ & $83.300^{\mathrm{ab}} \pm 8.3$ & $94.433^{\mathrm{a}} \pm 9.64$ \\
\hline
\end{tabular}

** Very significant differences in $\mathrm{P}<0.01$.

in all treatments $(\mathrm{P}<0.01)$. Based on Duncan's test results, it seems the fourth and first treatments have caused maximum (a rank) and minimum (c rank) averages in week 1 , respectively. The changes of fish weight, length, and specific growth rate are summarized in Figures 1-4. 
M Mohamadizadeh Kh et al., Effect of water current on growth of trout

Table 5 The results of fry ANOVA and Duncan's test in fifth week

\begin{tabular}{llllll}
\hline Studied indicators & Treatment & \multicolumn{5}{l}{} \\
\cline { 2 - 6 } & F5 & T1 & T2 & T3 & T4 \\
\hline Weight $(\mathrm{gr})$ & $7.604^{* *}$ & $18.7571^{\mathrm{c}} \pm 3.66$ & $25.8052^{\mathrm{bc}} \pm 5.75$ & $32.2331^{\mathrm{ab}} \pm 5.75$ & $40.2558^{\mathrm{a}} \pm 7.26$ \\
Length $(\mathrm{cm})$ & $2.617 \mathrm{~ns}$ & $12.1281^{\mathrm{b}} \pm 0.43$ & $12.2315^{\mathrm{ab}} \pm 0.48$ & $12.4044^{\mathrm{ab}} \pm 0.54$ & $13.0214^{\mathrm{a}} \pm 0.38$ \\
$\mathrm{SGR} \%$ & $12.696^{* *}$ & $1.5753^{\mathrm{b}} \pm 0.42$ & $2.3826^{\mathrm{ab}} \pm 0.52$ & $3.4561^{\mathrm{a}} \pm 0.69$ & $4.1440^{\mathrm{a}} \pm 0.52$ \\
$\mathrm{DGR} \%$ & $8.594^{* *}$ & $0.2883^{\mathrm{c}} \pm 0.12$ & $0.5701^{\mathrm{bc}} \pm 0.22$ & $0.9730^{\mathrm{ab}} \pm 0.35$ & $1.4651^{\mathrm{a}} \pm 0.41$ \\
$\mathrm{CF} \%$ & $13.811^{* *}$ & $1.0449^{\mathrm{c}} \pm 0.14$ & $1.3964^{\mathrm{b}} \pm 0.17$ & $1.6773^{\mathrm{ab}} \pm 0.11$ & $1.8103^{\mathrm{a}} \pm 0.18$ \\
$\mathrm{SR} \%$ & $15.568^{* *}$ & $41.633^{\mathrm{b}} \pm 8.35$ & $58.300^{\mathrm{b}} \pm 8.3$ & $77.773^{\mathrm{a}} \pm 12.72$ & $91.633^{\mathrm{a}} \pm 8.35$ \\
\hline
\end{tabular}

** Significant differences in $\mathrm{P}<0.01$, ${ }^{\mathrm{ns}}$ non significant differences, $\mathrm{T}=$ Treatment.

Table 6 Growth performance of trout treated in different water qualities 35 days post-experiment at $18 \pm 1.19^{\circ} \mathrm{C}$

\begin{tabular}{|c|c|c|c|c|c|}
\hline \multirow[t]{2}{*}{ Studied indicators } & \multicolumn{5}{|l|}{ Treatment } \\
\hline & F5 & $\mathrm{T} 1$ & $\mathrm{~T} 2$ & $\mathrm{~T} 3$ & $\mathrm{~T} 4$ \\
\hline Weight (gr) & $7.617 * *$ & $13.6915^{\mathrm{c}} \pm 1.82$ & $17.2561^{\mathrm{bc}} \pm 2.89$ & $20.0061^{\mathrm{ab}} \pm 2.38$ & $23.4302^{\mathrm{a}} \pm 3.08$ \\
\hline Length (cm) & $4.815^{*}$ & $9.9239^{\mathrm{b}} \pm 0.41$ & $10.3250^{\mathrm{ab}} \pm 0.39$ & $10.4920^{\mathrm{ab}} \pm 0.29$ & $10.9985^{\mathrm{a}} \pm 0.28$ \\
\hline SGR $\%$ & $7.059 * *$ & $3.4682^{\mathrm{c}} \pm 0.56$ & $4.3767^{\mathrm{b}} \pm 0.66$ & $5.0494^{\mathrm{a}} \pm 0.49$ & $5.5881^{\mathrm{a}} \pm 0.64$ \\
\hline DGR $\%$ & $7.605 * *$ & $0.3781^{\mathrm{c}} \pm 0.10$ & $0.5800^{b c} \pm 0.16$ & $0.7637^{\mathrm{ab}} \pm 0.16$ & $0.9929^{\mathrm{a}} \pm 0.20$ \\
\hline $\mathrm{CF} \%$ & $11.336^{*}$ & $1.4257^{\mathrm{b}} \pm 0.03$ & $1.5073^{\mathrm{ab}} \pm 0.04$ & $1.6124^{\mathrm{a}} \pm 0.02$ & $1.6174^{\mathrm{a}} \pm 0.06$ \\
\hline $\mathrm{SR} \%$ & $10.615^{* *}$ & $72.200^{c} \pm 6.73$ & $83.3066^{\mathrm{bc}} \pm 6.007$ & $91.0866^{\mathrm{ab}} \pm 5.09$ & $96.6533^{\mathrm{a}} \pm 4.42$ \\
\hline
\end{tabular}

** Significant differences in $\mathrm{P}<0.01$, * significant differences in $\mathrm{P}<0.05, \mathrm{~T}=$ Treatment.

\section{Discussion}

Concerning high fish density in this survey, water velocity was increased till dissolved $\mathrm{O}_{2}$ and $\mathrm{CO}_{2}$ increased and decreased during water fell, respectively. Summerfelt, Vinci \& Piedrahita (2000) showed that water oxygenation along with $\mathrm{CO}_{2}$ elimination are necessary factors in water reuse aquaculture systems. Our results implied that water velocity increment by outlet water reuse can also moderate these two factors. Clarke (2003) suggested that oxygen injection could increase fish raceway capacity, although our results showed that this matter could be done by water velocity increment through reuse of filtered outlet water. Water velocity increment increased mixing level between air and water. This could result in better dissolved $\mathrm{O}_{2}$ and $\mathrm{CO}_{2}$ balance in water. $\mathrm{CO}_{2}$ concentration could be bearable till $24 \mathrm{mg} \mathrm{L}^{-1}$ by rainbow trout in culture unit (Good, Davidson, Welsh, Snekvik \& Summerfelt 2010), although we never recorded such $\mathrm{CO}_{2}$ concentration during the experiment. At the same time, $\mathrm{CO}_{2}$ reduction in faster treatments was more evident. Ac- cording to Martins, Pistrin, Ende, Eding \& Verreth (2009) studies, solved and dissolved matter concentrations could be inhibitor factors in recirculation aquaculture systems, so their high amount could result in fry mortality. The results of this experiment showed that stocking density level could be differential based on water velocity in fry bearable limitations. In our study, water velocity increment was provided by outlet water reuse after physical filtration and aeration only. Our results justify Colt's findings who introduced water speed as an effective factor on reducing water pollution (Colt 2005). It was seen that water pollution occurred gradually and is reusable after aerating and total solid sediment (TSS) elimination. This issue has been supported by the previous findings (Summerfelt, Davidson, Waidrop, Tsukuda \& Williams 2004; Summerfelt \& Chris 2005; Stewart, Boardman \& Helfrich 2006). In this experiment, stocking density was more effective on fish growth and survival rate rather than water quality, and this matter has previously been reported (North, Ellis, Turnbull, Davis \& Bromage 2006; Person-Le Ruyet, Labbé, Le Bayon, Sévère, Le Roux, Le Delliou \& Quéméner 2008). These 
workers showed that desired water quality covers stocking density problems. In spite of stocking density increment, our results showed that water speed increment could reduce the stocking rate problems. At the same time, previous findings imply that fish density does not lead to a considerable effect on fish growth and survival (Lefrancois, Claireaux, Mercier \& Aubin 2001; North, Turnbull, Ellis, Porter, Migaud, Bron \& Bromage 2006). Roque d'orbcastel, Blancheton \& Belaud (2009) found that only water recirculation could supply fish survival without the necessity of water exchange. These results were justified by our findings in current water treatments, although this result was not observed in control treatment $(0 \mathrm{~cm} / \mathrm{s})$. In this experiment, weekly biometry showed some differences which could not be seen during the 35 day experiment. Duncan's test results justify that in the first week, the fourth treatment (10 $\mathrm{cm} / \mathrm{s}$ ) took the best rank (a) of growth factors excluding condition factor (CF). However, the reason was not distinctive and requires further studies. The weakest results were observed in static water treatment (control) while the fourth treatment $(10 \mathrm{~cm} / \mathrm{s})$ provided better results than the other treatments. It seems water velocity in our experiment was more important than water resource and its quality, so that water speed increment could affect water quality and adjust it to some extent.

This survey was done in laboratory condition, so its examination in farm condition could be a good point for future studies. It is recommended that other higher water speeds be examined in order to determine the limitation velocity for trout culture under laboratory condition.

\section{References}

Clarke M.L. (2003) Comparison of water quality, rainbow trout production and economics in oxygenated and aerated raceways. MSc thesis, Virginia Polytechnic Institute and State University, Blacksburg, VA.

Colt J. (2005) Water quality requirements for reuse systems. Aquacultural Engineering 34, 143-158.

Castell J. D. \& Tiews K. (1980) Report of the EIFAC, IUNS and ICES working group on the standardization of meth- odology in fish nutrition research. FAO, Technical Paper.

Good C., Davidson J., Welsh C., Snekvik K. \& Summerfelt S. (2010) The effects of carbon dioxide on performance and histopathology of rainbow trout (Oncorhynchus mykiss) in water recirculation aquaculture systems. Aquacultural Engineering 42, 51-56.

Jeffrey M. H. (1999) Trout production: feeds and feeding methods. SRAC publication. No. 223.

Lefrancois C., Claireaux G., Mercier C. \& Aubin J. (2001) Effect of density on the routine metabolic expenditure of farmed rainbow trout (Oncorhynchus mykiss). Aquaculture 195, 269- 277.

Martins C. I. M., Pistrin M. G., Ende S. S. W., Eding E. H. \& Verreth J. A. J. (2009) The accumulation of substances in Recirculating Aquaculture Systems (RAS) affects embryonic and larval development in common carp Cyprinus carpio. Aquaculture 291, 65-73.

North B. P., Ellis T., Turnbull J. F., Davis J. \& Bromage N. R. (2006) Stocking density practices of commercial UK rainbow trout farms. Aquaculture 259, 260-267.

North B. P., Turnbull J. F., Ellis T., Porter M. J., Migaud H., Bron J. \& Bromage N. R. (2006) The impact of stocking density on the welfare of rainbow trout (Oncorhynchus mykiss). Aquaculture 255, 466- 479.

Person-Le Ruyet J., Labbé L., Le Bayon N., Sévère A., Le Roux A., Le Delliou H. \& Quéméner L. (2008) Combined effects of water quality and stocking density on welfare and growth of rainbow trout (Oncorhynchus mykiss). Aquatic Living Resources 21, 185-195.

Roque d'orbcastel E., Blancheton J. P. \& Belaud A. (2009) Water quality and rainbow trout performance in a Danish Model Farm recirculating system:Comparison with a flow through system. Aquacultural Engineering 40, 135-143.

Sedgwick S. D. (1990) Trout farming handbook. Fishing News Book, Oxford, UK. 
Stewart N. T., Boardman G. D. \& Helfrich L. A. (2006) Treatment of rainbow trout (Oncorhynchus mykiss) raceway effluent using baffled sedimentation and artificial substrates. Aquacultural Engineering 35, 166-178.

Summerfelt S. T., Vinci B. J. \& Piedrahita R. H. (2000) Oxygenation and carbone dioxide control in water reuse systems. Aquacultural Engineering 22, 87-108.

Summerfelt S. T., Davidson J. W., Waidrop T. B.,Tsukuda S. M. \& Williams J. B. (2004) A partial-reuse system for cold water aquaculture. Aquacultural Engineering 31, 157181.

Summerfelt R. C. \& Chris R. P. (2005) Solids removal in a recirculating aquaculture system where the majority of flow bypasses the microscreen filter. Aquacultural Engineering 33, 214-224.

Willoughby S. (1999) Manual of Salmonid farming. Fishing News Books, Blackwell Science, Oxford. 


\title{
تأثير جريان آب بر روى برخى بارامترهاى رشد و كيفيت آب در برورش ماهيط ماهى قزل

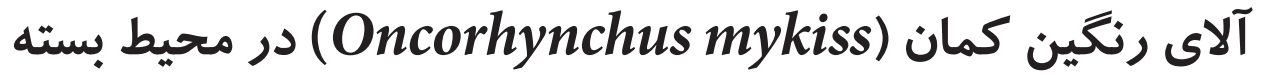

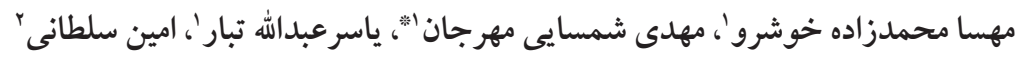

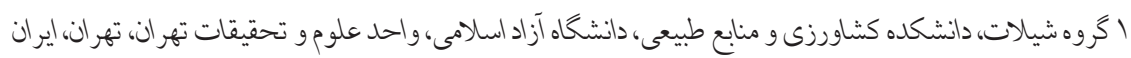

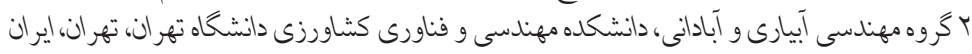

در اين مطالعه اثرات افزايش جريان سرعت آب برماهى قزل آلاى انگشت قد در محيط بسته يرورشى در مدت هـ روز مورد بررسى قرار گرفت. ابتدا

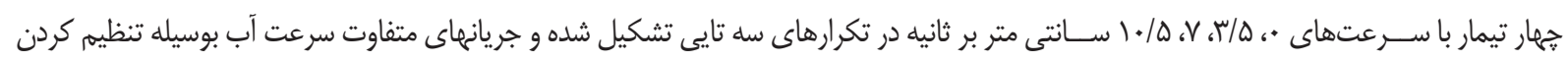

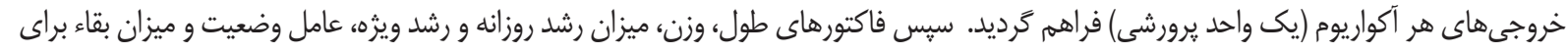

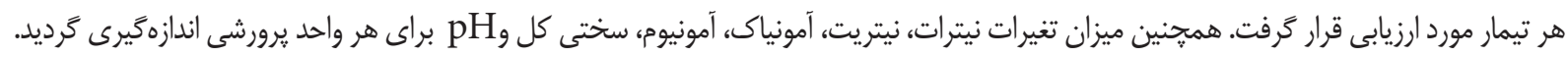

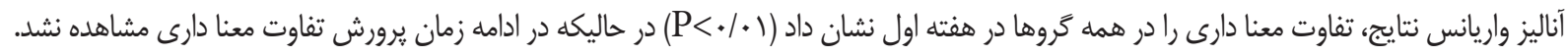

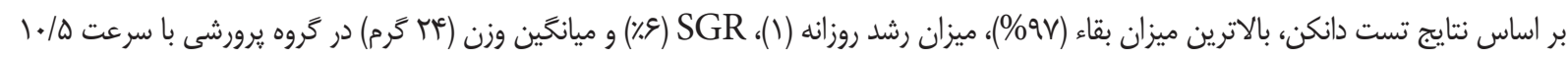
سانتى متر بر ثانيه بدست آمد. وازههاى كليدى: قزل الاى رنخين كمان، برامترهاى رشد، آب خروجى، سيستم بسته، سرعت جريان. drshamsaie@gmail.com : 